\title{
Hepatic mitochondrial energetics during catch-up fat after caloric restriction
}

\author{
Raffaella Crescenzo $^{\mathrm{a}}$, Francesca Bianco $^{\mathrm{a}}$, Italia Falcone ${ }^{\mathrm{a}}$, Marina Prisco ${ }^{\mathrm{b}}$, \\ Abdul G. Dulloo ${ }^{\text {, }}$, Giovanna Liverini ${ }^{\mathrm{a}}$, Susanna Iossa ${ }^{\mathrm{a}, *}$ \\ ${ }^{a}$ Section of Physiology, Department of Biological Sciences, University of Naples, via Mezzocannone 8, I-80134, Napoli, Italy \\ ${ }^{\mathrm{b}}$ Section of Evolutionary and Comparative Biology, Department of Biological Sciences, University of Naples, via Mezzocannone 8, I-80134, Napoli, Italy \\ ${ }^{\mathrm{c}}$ Division of Physiology, Department of Medicine, University of Fribourg, Chemin du Musee 5, CH-1700, Fribourg, Switzerland
}

\begin{abstract}
The objective of the study was to investigate whether changes in liver mitochondrial energetics could underlie the enhanced energetic efficiency that drives accelerated body fat recovery (catch-up fat) during refeeding after caloric restriction. Rats were subjected to caloric restriction (50\% of ad libitum intake) for 15 days and then refed for 1 or 2 weeks on an amount of chow equal to that of controls matched for weight at the onset of refeeding. Whole-body metabolism was characterized by energy balance and body composition determinations as well as by indirect calorimetric measurements of 24-hour energy expenditure, substrate oxidation, and whole-body de novo lipogenesis estimated from nonprotein respiratory quotient. Hepatic mitochondrial energetics were determined from measurements of liver mitochondrial mass, respiratory capacities, and proton leak (both basal and fatty acid stimulated), whereas hepatic oxidative status was assessed from measurements of hepatic mitochondrial lipid peroxidation, aconitase, and superoxide dismutase activity. Furthermore, hepatic lipogenic capacity was determined from assays of fatty acid synthase activity. Compared with controls, isocalorically refed rats showed an elevated energetic efficiency and body fat gain over both week 1 and week 2 of refeeding, as well as a lower 24-hour energy expenditure and higher rates of whole-body de novo lipogenesis at the end of both week 1 and week 2 of refeeding. Analysis of the liver revealed that after 1 week (but not after 2 weeks) of refeeding, the mitochondrial mass (but not mitochondrial density) was lower in refed rats than in controls, associated with higher state 3 mitochondrial respiratory capacity, increased superoxide dismutase activity, as well as higher fatty acid synthase activity. These results suggest that, although at the whole-body level elevations in energy efficiency and de novo lipogenesis are coordinated toward catch-up fat, the overall hepatic mitochondrial energetic status during refeeding is more consistent with a contributory role of the liver in the enhanced de novo lipogenic machinery during catch-up fat rather than in the energy-conservation mechanisms (elevated energetic efficiency) that spare energy for catch-up fat.
\end{abstract}

\section{Introduction}

In response to reduced food intake, energy expenditure falls to an extent that is often greater than that predicted by loss of tissues and that is partly attributed to an increase in metabolic efficiency [1]. This adaptive reduction in thermogenesis that allows the organism to spare energy (thus slowing the rate of depletion of lean and fat stores) during

* Corresponding author. Dipartimento delle Scienze Biologiche, Sezione di Fisiologia,Via Mezzocannone 8, I-80134 Napoli, Italy. Tel.: +39081 2535088; fax: +39081 2535090 .

E-mail address: susiossa@unina.it (S. Iossa). caloric restriction also persists for some time upon refeeding [2], thus allowing rapid recovery of body fat stores. This thrifty metabolism directed at accelerating fat recovery (or catch-up fat), which has evolved in a context of periodic food shortage, contributes to the failure of dieting in long-term management of overweight and obesity in modern societies. In addition, human and animal studies have implicated the phenomenon of preferential catch-up fat in the link between catch-up growth (after earlier growth retardation) and a high predisposition to central obesity, impaired glucose tolerance, and cardiovascular disease later in life $[3,4]$.

The reduced energy expenditure and adaptive suppression of thermogenesis elicited by caloric restriction are the 
outcomes of metabolic changes in single organs and tissues, most likely involving those that are major contributors of daily metabolic rate [5]. In fact, recent studies have suggested that skeletal muscle is involved in the suppression of thermogenesis that underlies the high metabolic efficiency for catch-up fat. Rats showing catch-up fat exhibit diminished subsarcolemmal mitochondrial mass and oxidative capacity in skeletal muscle [6]. In addition, in vivo insulin-stimulated glucose utilization has been shown to be lower in skeletal muscle but higher in adipose tissue of refed animals showing catch-up fat, thereby suggesting a redistribution of glucose utilization away from skeletal muscle toward de novo lipogenesis and fat storage in adipose tissue [7].

Another important tissue/organ contributor to wholebody energy consumption is the liver. It has a high metabolic activity [5] contributing about a quarter of resting metabolic rate, and it is of central importance in the orchestration of the supply of energy substrates to different tissues. Taking into account that the main cellular compartment involved in the control of cellular energy handling is represented by the mitochondria, the aim of the present study was to investigate the extent to which changes in hepatic mitochondrial mass, respiratory performance, and efficiency could underlie the suppressed thermogenesis that drives catch-up fat during refeeding after caloric restriction. To this end, we measured mitochondrial state 3 and state 4 respiratory capacities in homogenates and isolated organelles by using nicotinamide adenine dinucleotide (NAD) and flavin adenine dinucleotide (FAD) substrates, as well as mitochondrial proton leak in liver from rats showing catch-up fat. Mitochondrial oxidative damage and antioxidant defense were also determined from measurements of lipid peroxidation, aconitase, and superoxide dismutase (SOD) specific activity. Before these studies centered on hepatic mitochondria, we performed a complete energy balance study so as to obtain a more detailed time course of the adaptive modifications of energy expenditure during catch-up fat in response to isocaloric refeeding after caloric restriction. Whole-body metabolic characteristics were obtained by measuring 24-hour oxygen consumption $\left(\mathrm{VO}_{2}\right)$, carbon dioxide production $\left(\mathrm{VCO}_{2}\right)$, and nonprotein respiratory quotient (NPRQ) using indirect calorimetry together with urinary nitrogen analysis.

\section{Methods and materials}

\subsection{General study design}

Male Sprague-Dawley rats were adapted to room and cage environments for 1 week before the start of the experiment. The rats were caged singly in a temperaturecontrolled room $\left(23^{\circ} \mathrm{C} \pm 1{ }^{\circ} \mathrm{C}\right)$ with a 12-hour light-dark cycle and were maintained on a commercial pelleted chow diet (Mucedola, Settimo Milanese, Italy) consisting, by energy, of $29.0 \%$ protein, $60.4 \%$ carbohydrates, and $10.6 \%$ fat and had free access to tap water. The experiments were conducted after this period of adaptation in rats selected on the basis of body weight being within $\pm 5 \mathrm{~g}$ of the mean body weight. Animals used in the present studies were maintained in accordance with Italian Health Ministry regulations and guidelines for the care and use of laboratory animals.

\subsection{Experimental design}

The experiments were performed using a design similar to that previously described in establishing a rat model that allows the adaptive reduction in energy expenditure (ie, suppressed thermogenesis) specific for accelerating fat recovery (catch-up fat) to be studied in the absence of confounding variables, such as differential food intake and differential rates of protein gain, on energy expenditure [2,4]. In brief, groups of 7-week-old rats with a mean body weight of $232 \pm 5 \mathrm{~g}$ were food restricted for 14 days at approximately $50 \%$ of their ad libitum food intake. This reduction in food intake was able to arrest growth, as shown by the fact that at the end of this period the mean body weight of rats $(229 \pm 4 \mathrm{~g})$ was not significantly different from the initial value. After this period, the semistarved animals were refed an amount of chow diet equal to the ad libitum food intake of control rats matched for weight at the onset of refeeding. Under these conditions, the refed animals show similar gain in lean mass, but greater gain in body fat than controls during 2 to 3 weeks of isocaloric refeeding. In the experiments described here, changes in body composition and energy content were determined from subgroups of rats $(n=4)$ killed at onset of refeeding (ie, at end of the semistarvation period) and after either 1 week or 2 weeks of isocaloric refeeding.

\subsection{Twenty-four-hour $\mathrm{VO}_{2}, \mathrm{VCO}_{2}$, urinary nitrogen, and $N P R Q$}

The day before the sacrifice of the animals at week 1 or 2 of refeeding, 24-hour $\mathrm{VO}_{2}$ and $\mathrm{VCO}_{2}$ were recorded with a monitoring system (Panlab, Cornella, Barcelona, Spain) composed of a 4-chamber indirect open-circuit calorimeter, designed for the continuous monitoring of up to 4 rats simultaneously. Measurements were performed every 15 minutes for 3 minutes in each cage. Urine was collected for the whole (24-hour) period; total volume was measured, and a portion of it was used for the determination of urinary nitrogen levels. In particular, urine urea nitrogen was measured by an enzymatic colorimetric method (FAR, Settimo di Pescantina, Verona, Italy); the values were then extrapolated to total urine nitrogen by multiplying by 1.25 (to take into account nitrogen excreted as uric acid and ammonia) and used for calculation of 24-hour NPRQ.

\subsection{Determination of body composition, liver lipid content, and fatty acid synthase activity}

At the end of the experimental period, the animals were killed by decapitation, the liver was harvested, the guts were cleaned of undigested food, and the carcasses were then autoclaved. After dilution (1:2 distilled water) and subse- 
quent homogenization of the carcasses with a Polytron homogenizer (Kinematica, Lucerne, Switzerland), the resulting homogenates were frozen at $-20^{\circ} \mathrm{C}$ until the day of measurements. Duplicate samples of the homogenized carcass were analyzed for energy content by bomb calorimetry. To take into account the energy content of the liver, tissue samples were dried; and the energy content was then measured with the bomb calorimeter. Total body fat content was measured by the extraction method of Folch et al [8]. Water content was determined by drying samples in an oven maintained at $70^{\circ} \mathrm{C}$ for 48 hours. Body protein was determined from a general formula relating energy derived from fat, total energy value of the carcass, and energy derived from protein [2]; the caloric values for body fat and protein were taken as 39.2 and $23.5 \mathrm{~kJ} / \mathrm{g}$, respectively. Samples of liver tissue were homogenized with distilled water (final volumes equal to twice the sample weight). Aliquots of the homogenates were analyzed for lipid content by the method of Folch et al [8]. Fatty acid synthase (FAS) activity was measured according to the protocol described by Pénicaud et al [9].

\subsection{Energy balance measurements}

Energy balance measurements were conducted by the comparative carcass technique over 1 and 2 weeks of refeeding, as detailed previously [10]. Briefly, during the experimental period, metabolizable energy (ME) intake was obtained by subtracting the energy measured in feces and urine from the gross energy intake, determined from daily food consumption and gross energy density of the diet. Body energy, protein, and fat gained during the first week, the second week, or the whole 2-week period were calculated as the difference between the final and initial content of body energy, protein, and fat. Energetic efficiency was calculated as the percentage of body energy retained per ME intake. Finally, energy expenditure was determined as the difference between ME intake and energy gain. The cost of storage was determined taking into account that the energy loss in storing $1 \mathrm{~kJ}$ protein is $1.25 \mathrm{~kJ}$ [10]. As for the energy cost for fat deposition, in animals consuming a diet with a high percentage of carbohydrate, such as the diet used in the present study, almost all of the body fat will be synthesized de novo from glucose with a relatively high energy cost $(0.36$ $\mathrm{kJ} / \mathrm{kJ}$ fat deposited) [10]. Net energy expenditure (NEE) was obtained from energy expenditure by subtracting the cost of protein and lipid storage.

\subsection{Mitochondrial ultrastructural observations and mitochondrial mass determination}

Liver samples were fixed in a mixture of $4 \%$ paraformaldehyde and $2.5 \%$ glutaraldehyde for 3 hours at room temperature in $0.1 \mathrm{~mol} / \mathrm{L}$ cacodylate buffer $(\mathrm{pH} 7.4)$, postfixed for 1 hour in $2 \% \mathrm{OsO}_{4}$ in cacodylate buffer at $4^{\circ} \mathrm{C}$, dehydrated and embebbed in Epon (Electron Microscopy Sciences, Hatfield, PA). Ultrathin sections were cut on a diamond knife, collected on Formvar (Electron Microscopy Sciences)-coated copper grids, stained with uranyl acetate and lead citrate, and examined with a Philips (Amsterdam, the Netherlands) 208S transmission electron microscope. Mitochondrial numerical density was calculated according to Gundersen et al [11].

Mitochondrial mass was indirectly assessed by measuring the activity of a mitochondrial marker enzyme, citrate synthase (CS), in liver homogenate and isolated mitochondria, according to Srere [12]. The CS activity, measured in the homogenate and expressed per gram wet liver, reflects the product of mitochondrial mass and specific activity of the CS enzyme. To determine CS specific activity, measurements were made in isolated mitochondria and the results were expressed per milligram of mitochondrial proteins. Finally, mitochondrial mass, expressed as milligrams per gram wet liver, was calculated as the ratio between CS activity in the homogenate and isolated mitochondria.

Mitochondrial mass was also evaluated by Western blot analysis of cytochrome $c$. To this end, homogenate samples were denatured in a buffer $(60.0 \mathrm{mmol} / \mathrm{L}$ Tris [pH 6.8], $10 \%$ saccarose, $2 \%$ sodium dodecyl sulfate [SDS], 4\% $\beta$ mercaptoethanol) and loaded onto a $12 \%$ SDS polyacrylamide gel. After the run in electrode buffer $(50 \mathrm{mmol} / \mathrm{L}$ Tris [pH 8.3], $384 \mathrm{mmol} / \mathrm{L}$ glycine, $0.1 \%$ SDS), these gels were transferred onto polyvinylidene difluoride membranes (Immobilon-P; Millipore, Billerica, MA) at $0.8 \mathrm{~mA} / \mathrm{cm}^{2}$ for 90 minutes. The membranes were preblocked in blocking buffer (phosphate-buffered saline [PBS], 5\% milk powder, $0.5 \%$ Tween 20 ) for 1 hour and then incubated overnight at $4^{\circ} \mathrm{C}$ with a mouse monoclonal antibody for cytochrome $c$ (Biomol, Plymouth Meeting, PA) diluted 1:100 in blocking buffer. Membranes were washed 3 times 12 minutes in PBS/ $0.5 \%$ Tween 20 and 3 times 12 minutes in PBS and then incubated for 90 minutes at room temperature with an antimouse, alkaline phosphatase-conjugated secondary antibody (Sigma-Aldrich, St Louis, MO). The membranes were washed as described above, rinsed in distilled water, and incubated at room temperature with a chemiluminescent substrate, CDP-Star (Sigma-Aldrich). To normalize the specific signal in each lane, actin was detected as above using a rabbit polyclonal antibody (Sigma-Aldrich) and an anti-rabbit, alkaline phosphatase-conjugated secondary antibody (Promega, Madison, WI). Data detection was carried out by exposing autoradiography films (Kodak; Eastman Kodak, Rochester, NY) to the membranes. Quantification of signals was carried out by Un-Scan-It gel software (Silk Scientific, Orem, UT).

\subsection{Mitochondrial respiration, proton leak, lipid peroxidation, aconitase, and SOD specific activity}

Liver homogenates and isolated mitochondria were prepared as previously reported [13]. Control experiments of enzymatic and electron microscopy characterization have shown that our isolation procedure (centrifugation at $3000 g_{\mathrm{av}}$ 
for 10 minutes) results in a cellular fraction essentially constituted by mitochondria.

Oxygen consumption was measured polarographically with a Clark-type electrode (Yellow Springs Instruments, Yellow Springs, $\mathrm{OH})$ at a temperature of $30^{\circ} \mathrm{C}$. Samples of homogenates or isolated mitochondria were incubated in a medium containing $80 \mathrm{mmol} / \mathrm{L} \mathrm{KCl}, 50 \mathrm{mmol} / \mathrm{L}$ HEPES, 5 $\mathrm{mmol} / \mathrm{L} \mathrm{K}_{2} \mathrm{HPO}_{4}, 1 \mathrm{mmol} / \mathrm{L}$ EGTA, and $0.1 \%$ (wt/vol) fatty acid-free bovine serum albumin $(\mathrm{pH} 7.0)$ and allowed to oxidize their endogenous substrates for 3 minutes. The substrates used were $10 \mathrm{mmol} / \mathrm{L}$ glutamate $+2.5 \mathrm{mmol} / \mathrm{L}$ malate, or $40 \mu \mathrm{mol} / \mathrm{L}$ palmitoyl-coenzyme A $(\mathrm{CoA})+2$ $\mathrm{mmol} / \mathrm{L}$ carnitine $+2.5 \mathrm{mmol} / \mathrm{L}$ malate. State 3 oxygen consumption was measured in the presence of $0.3 \mathrm{mmol} / \mathrm{L}$ adenosine diphosphate. State 4 respiration was obtained in the presence of oligomycin $(4 \mu \mathrm{g} / \mathrm{mL})$ in the homogenates or in the absence of adenosine diphosphate in isolated mitochondria. Respiratory control ratio (RCR) was calculated according to Estabrook [14].

Proton leak kinetics were obtained by parallel measurements of mitochondrial oxygen consumption by using a Clark-type electrode; and membrane potential, by using safranin O and a JASCO (Tokyo, Japan) dual-wavelength spectrophotometer $(511-533 \mathrm{~nm})$ as previously reported [6].

Lipid peroxidation was determined according to Fernandes et al [15]. The specific activities of active aconitase, total aconitase, and SOD were measured as previously described [16].

\subsection{Statistical analysis}

Data are given as means \pm standard error of the mean (SEM). Statistical analyses were performed by 2-way analysis of variance (ANOVA) for main effects and interactions, followed by Bonferroni post hoc test. Probability values less than .05 were considered to indicate a significant difference. All analyses were performed using GraphPad Prism 4 (GraphPad Software, San Diego, CA).

\subsection{Materials}

All chemicals used were of analytical grade and were purchased from Sigma.

\section{Results}

\subsection{Energy balance, body composition, nutrient partitioning, and de novo lipogenesis}

Table 1 reports data on body composition at the end of semistarvation and after 1 and 2 weeks of isocaloric refeeding. At the end of the semistarvation period (time 0 of refeeding), the semistarved and control rats exhibited similar body weight and proteins and slightly higher body water. By contrast, body energy and lipids were found to be lower in semistarved rats compared with controls. These significantly lower values were no longer present after week 1 of isocaloric refeeding, when refed rats had similar body composition to control rats. At the end of week 2 of refeeding, refed rats showed significantly higher body energy $(+8 \%)$ and lipids $(+17 \%)$ compared with controls.

Table 2 shows results on energy balance in refed and control rats during week 1 , week 2 , and over the entire 2 -week period. After week 1 of refeeding, the refed rats showed significantly higher energy and lipid gain, together with significantly lower energy expenditure and higher gross efficiency. During week 2 of refeeding, the above between-group differences were less marked; but they nonetheless reached statistical significance for lipid gain and energy expenditure. When energy balance was analyzed over the entire 2-week period, refed rats showed significantly higher energy gain, lipid gain, and gross efficiency and significantly lower energy expenditure compared with controls. No significant between-group differences were found for protein gain over week 1 , week 2 , or over the entire 2-week period of refeeding.

Table 3 reports data on energy, lipid, and protein partitioning in control and refed rats. As for energy partitioning, an estimate of the cost of lipid deposition gave significantly higher values of energy expended for storing lipids during week 1 and week 2, as well as over the entire 2-week period, for refed rats. Net energy expenditure, both as absolute value and expressed as percentage of ME intake, was significantly lower during week 1 and week 2 , as well as during the entire 2-week period, although to a lesser extent in week 2 than in week 1. Lipid partitioning between oxidation and storage was drastically modified by refeeding, so that refed rats always exhibited significantly higher values

Table 1

Body composition at the beginning and after 1 and 2 weeks of refeeeding

\begin{tabular}{|c|c|c|c|c|c|c|}
\hline & \multicolumn{2}{|c|}{$0 \mathrm{wk}$} & \multicolumn{2}{|c|}{$1 \mathrm{wk}$} & \multicolumn{2}{|c|}{$2 \mathrm{wk}$} \\
\hline & Control & Refed & Control & Refed & Control & Refed \\
\hline Body weight, g & $225 \pm 5$ & $229 \pm 4$ & $279 \pm 6$ & $296 \pm 7$ & $313 \pm 6$ & $338 \pm 4$ \\
\hline Body energy, $\mathrm{kJ} / \mathrm{g}$ & $8.1 \pm 0.2$ & $6.6 \pm 0.2^{\dagger}$ & $8.3 \pm 0.2$ & $8.2 \pm 0.2$ & $8.7 \pm 0.1$ & $9.4 \pm 0.1^{*}$ \\
\hline Body lipids, \% & $9.5 \pm 0.2$ & $5.9 \pm 0.1^{\dagger}$ & $9.7 \pm 0.3$ & $9.9 \pm 0.4$ & $11.0 \pm 0.3$ & $12.9 \pm 0.3^{\dagger}$ \\
\hline Body proteins, $\%$ & $18.2 \pm 0.5$ & $18.3 \pm 0.4$ & $18.9 \pm 1.0$ & $18.4 \pm 0.6$ & $18.6 \pm 0.4$ & $18.1 \pm 0.7$ \\
\hline Body water, \% & $67.8 \pm 0.6$ & $68.5 \pm 0.7$ & $67.7 \pm 0.6$ & $66.2 \pm 0.8$ & $66.3 \pm 0.6$ & $64.2 \pm 0.5$ \\
\hline
\end{tabular}

Values are the means \pm SEM of 6 different experiments.

* $P<.05$ compared with control (2-way ANOVA followed by Bonferroni posttest).

${ }^{\dagger} P<.001$ compared with control (2-way ANOVA followed by Bonferroni posttest). 
Table 2

Energy balance in refed and control rats

\begin{tabular}{|c|c|c|c|c|c|c|}
\hline & \multicolumn{2}{|c|}{ 1st wk } & \multicolumn{2}{|c|}{ 2nd wk } & \multicolumn{2}{|c|}{ 2-wk period } \\
\hline & Control & Refed & Control & Refed & Control & Refed \\
\hline ME intake, kJ & $2374 \pm 60$ & $2302 \pm 38$ & $2156 \pm 136$ & $2114 \pm 63$ & $4530 \pm 121$ & $4415 \pm 39$ \\
\hline Energy gain, $\mathrm{kJ}$ & $532 \pm 58$ & $917 \pm 84^{*}$ & $508 \pm 55$ & $731 \pm 112$ & $1011 \pm 71$ & $1655 \pm 83^{\dagger}$ \\
\hline Lipid gain, $\mathrm{kJ}$ & $248 \pm 46$ & $614 \pm 58^{\dagger}$ & $368 \pm 50$ & $566 \pm 58^{*}$ & $569 \pm 50$ & $1186 \pm 58^{\dagger}$ \\
\hline Protein gain, $\mathrm{kJ}$ & $306 \pm 65$ & $300 \pm 54$ & $164 \pm 42$ & $165 \pm 42$ & $463 \pm 36$ & $465 \pm 65$ \\
\hline Energy expenditure, kJ & $1841 \pm 55$ & $1384 \pm 111^{\dagger}$ & $1688 \pm 59$ & $1383 \pm 80^{*}$ & $3519 \pm 87$ & $2761 \pm 51^{\dagger}$ \\
\hline Gross efficiency, $\%$ & $22 \pm 2$ & $40 \pm 4^{\dagger}$ & $25 \pm 3$ & $34 \pm 2$ & $22 \pm 2$ & $37 \pm 2^{\dagger}$ \\
\hline
\end{tabular}

Values are the means \pm SEM of 6 different experiments.

$* P<.05$ compared with control (2-way ANOVA followed by Bonferroni posttest).

$\dagger P<.001$ compared with control (2-way ANOVA followed by Bonferroni posttest)

for lipid gain/lipid intake, although between-group differences were greater in week 1 than week 2 . No significant differences were found in protein partitioning over both week 1 and week 2 of refeeding.

Fig. 1A shows the results of 24-hour monitoring of $\mathrm{VO}_{2}$ and $\mathrm{VCO}_{2}$ in refed and control animals. A significant decrease in mean 24-hour $\mathrm{VO}_{2}$ and $\mathrm{VCO}_{2}$ and a significant increase in NPRQ (Fig. 1A) were found after week 1 and week 2 of refeeding. The rates of de novo lipogenesis, calculated using NPRQ values according to the method of Elia and Livesey [17], were found to be significantly higher in refed rats than in controls after both week 1 and week 2 of refeeding.

\subsection{Hepatic lipid content and FAS activity}

Hepatic FAS activity was significantly higher after week 1 of refeeding, whereas no differences were found in hepatic lipid content at week 1 or week 2 of refeeding (Fig. 1B).

\subsection{Hepatic mitochondrial mass, energetics, and oxidative status}

Measurements of CS activity indicate that the enzyme activity, measured in the homogenate and expressed per gram wet liver (Fig. 2A), was significantly lower in refed rats after week 1 of refeeding, whereas no differences were found after week 2. However, CS specific activity, expressed per milligram mitochondrial protein (Fig. 2B), was similar in refed and control rats. Therefore, mitochondrial mass (Fig. 2C), calculated as the ratio between CS activity in the homogenate and isolated mitochondria, was significantly lower in refed rats after week 1 of refeeding, but returned to control values after week 2. Accordingly, homogenate cytochrome $c$ protein content evaluated by Western blot analysis was significantly lower in refed rats after 1 week but not after 2 weeks of refeeding (Fig. 2E). No differences in mitochondrial numerical density were found after week 1 or week 2 in refed rats compared with controls (Fig. 2D).

Liver mitochondrial state 3 and 4 respiratory capacities were first measured in homogenates to take into account changes in protein mass and capacity of mitochondria of the whole tissue due to refeeding (Table 4). Using NAD-linked substrate (glutamate + malate) and lipid substrate (palmitoyl$\mathrm{CoA}+$ carnitine + malate), no significant differences were found in respiratory capacities in refed rats compared with controls.

Respiratory capacities were also measured in isolated liver mitochondria to take into account changes in specific capacity due to refeeding (Table 4). State 3 respiratory capacity significantly increased in refed rats after week 1 of refeeding, whereas no differences were found after week 2 .

Mitochondrial proton leak was assessed by titration of steady-state respiration rate as a function of mitochondrial membrane potential in liver mitochondria. This titration curve is an indirect measurement of proton leak because steady-state oxygen consumption rate (ie, proton efflux rate)

Table 3

Partitioning of ME, lipid, and protein intake in refed and control rats

\begin{tabular}{|c|c|c|c|c|c|c|}
\hline & \multicolumn{2}{|c|}{ 1st wk } & \multicolumn{2}{|c|}{ 2nd wk } & \multicolumn{2}{|c|}{ 2-wk period } \\
\hline & Control & Refed & Control & Refed & Control & Refed \\
\hline Cost of lipids, $\mathrm{kJ}$ & $89 \pm 7$ & $221 \pm 21^{\dagger}$ & $132 \pm 13$ & $204 \pm 11^{*}$ & $205 \pm 18$ & $427 \pm 33^{\dagger}$ \\
\hline Cost of proteins, $\mathrm{kJ}$ & $382 \pm 81$ & $375 \pm 68$ & $205 \pm 33$ & $206 \pm 11$ & $579 \pm 41$ & $581 \pm 41$ \\
\hline NEE, kJ & $1370 \pm 56$ & $788 \pm 56^{\dagger}$ & $1351 \pm 68$ & $973 \pm 34^{*}$ & $2735 \pm 76$ & $1752 \pm 66^{\dagger}$ \\
\hline NEE/ME intake, \% & $57.9 \pm 2.2$ & $33.8 \pm 2.1^{\dagger}$ & $62.7 \pm 1.8$ & $46.0 \pm 2.1^{*}$ & $60.4 \pm 3.0$ & $39.8 \pm 2.8^{\dagger}$ \\
\hline Lipid gain/lipid intake, $\%$ & $97.6 \pm 16.4$ & $252.9 \pm 25.2^{\dagger}$ & $167.2 \pm 26.5$ & $250.6 \pm 46.0^{*}$ & $119.0 \pm 11.4$ & $252.8 \pm 17.9^{\dagger}$ \\
\hline Protein gain/protein intake, $\%$ & $44.4 \pm 2.1$ & $45.4 \pm 3.2$ & $26.2 \pm 2.3$ & $26.9 \pm 1.3$ & $35.2 \pm 2.8$ & $36.4 \pm 2.3$ \\
\hline
\end{tabular}

Values are the means \pm SEM of 6 different experiments.

$* P<.05$ compared with control (2-way ANOVA followed by Bonferroni posttest).

${ }^{\dagger} P<.001$ compared with control (2-way ANOVA followed by Bonferroni posttest). 

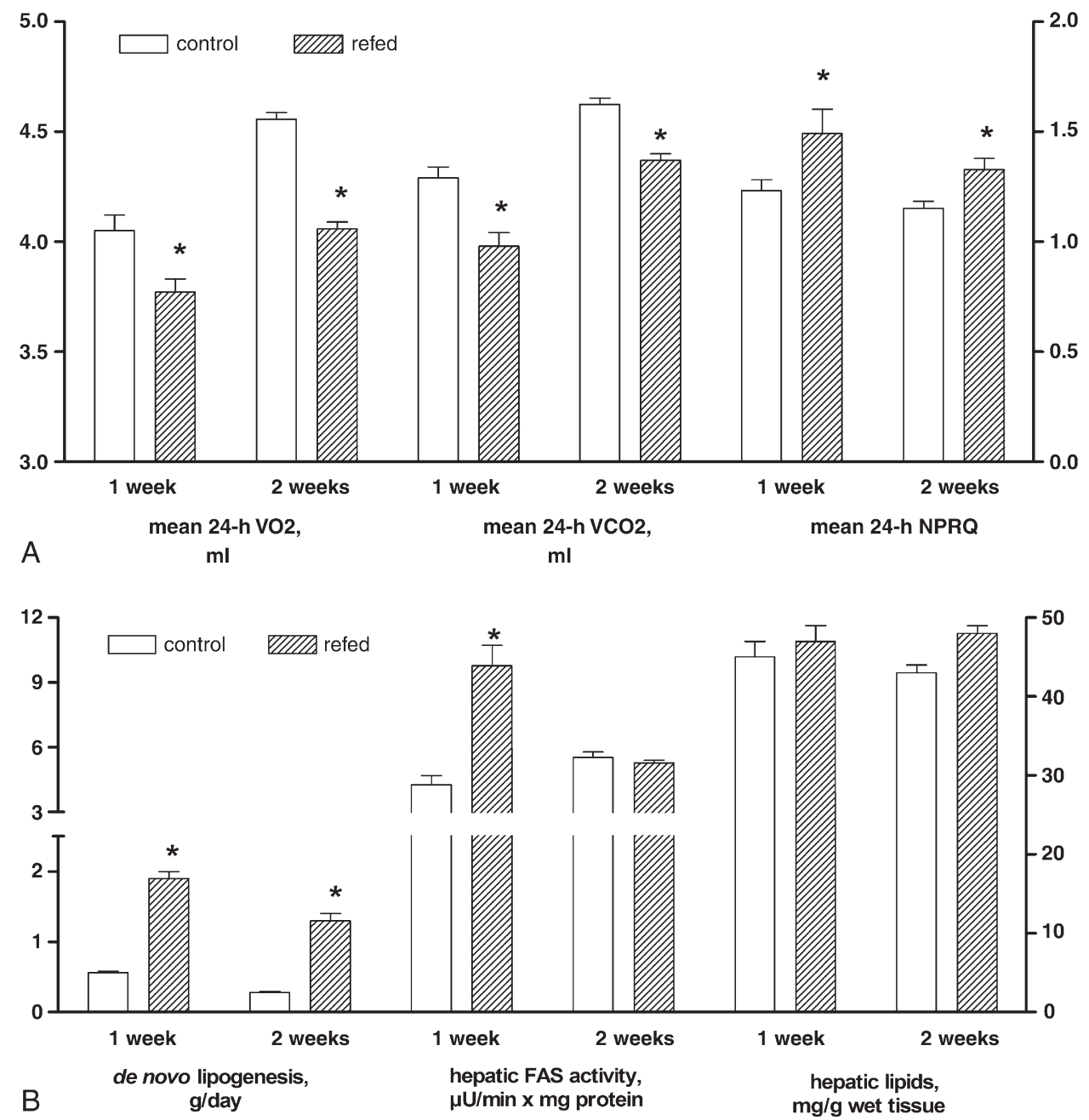

Fig. 1. Mean 24-hour $\mathrm{VO}_{2}, \mathrm{VCO}_{2}$, and NPRQ (A), as well as rates of de novo lipogenesis, hepatic FAS activity, and hepatic lipids (B) after 1 and 2 weeks of refeeding. Results are the means \pm SEM of 6 different experiments. $* P<.05$ compared with control (2-way ANOVA followed by Bonferroni posttest).

in nonphosphorylating mitochondria is equivalent to proton influx rate due to proton leak. Fig. 3 shows that proton leak did not differ in mitochondria from refed and controls after week 1 and week 2 of refeeding.

To assess oxidative damage and antioxidant defense in liver mitochondria, lipid peroxidation, aconitase, and SOD specific activities were tested. The results show that refeeding for 1 and 2 weeks did not elicit significant changes in oxidative damage, whereas a significant increase in antioxidant defenses was found only after week 1 of refeeding (Table 5).

\section{Discussion}

Using a previously established rat model of semistarvation-refeeding in which accelerated fat deposition (catch-up fat) is driven solely by reduced energy expenditure and not by hyperphagia $[2,4]$, we previously reported that diminished skeletal muscle mitochondrial mass and function, specifically in the subsarcolemmal mitochondrial compartment, contribute to the high energetic efficiency for catch-up fat [6]. The studies reported here, centered upon the hypothesis that the liver may also be an important site for the diminished thermogenesis that underlies catch-up fat, suggest however that altered hepatic mitochondrial energetics may be more relevant to an enhancement in hepatic de novo lipogenic machinery for catch-up fat rather than to energy-conservation mechanisms that spare energy for catch-up fat.

\subsection{Further characterization of thrifty metabolism driving catch-up fat}

The data on energy balance and body composition presented here strongly support the importance of an adaptive increase in energetic efficiency in the replenishment of body fat stores, albeit this enhancement in energetic efficiency was more pronounced in the first week than in the second week of refeeding. The high metabolic efficiency 

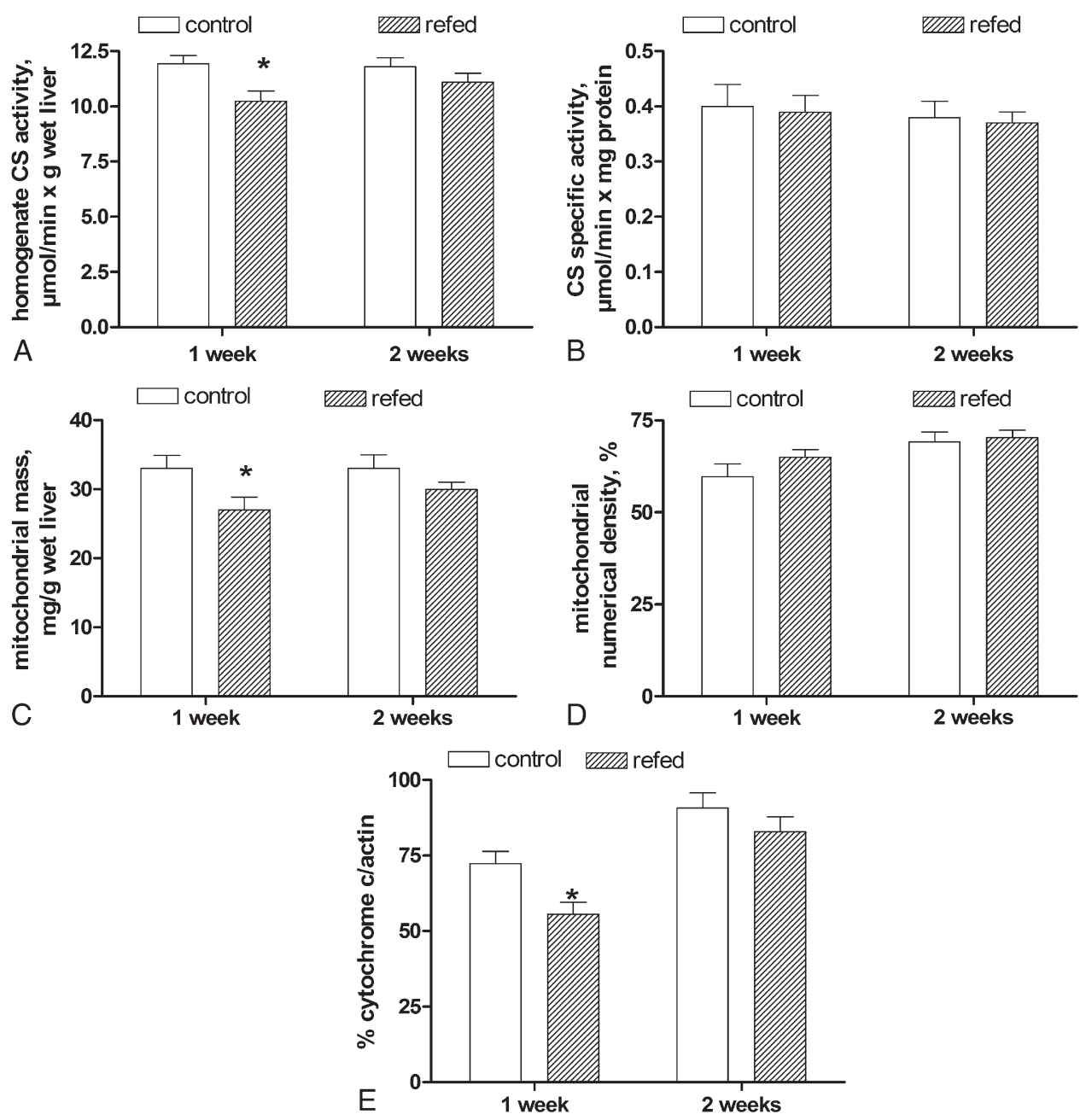

Fig. 2. Homogenate CS activity per gram wet liver (A), CS specific activity per milligram mitochondrial protein (B), mitochondrial mass expressed as milligrams per gram wet liver (C), percentage mitochondrial numerical density (D), and homogenate cytochrome $c$ protein content (E) after 1 and 2 weeks of refeeding. Results are the means \pm SEM of 6 different experiments. ${ }^{*} P<.05$ compared with control (2-way ANOVA followed by Bonferroni posttest).

underlying the catch-up fat phenomenon is unlikely due to differential meal pattern in refed and control rats (gorging vs nibbling, respectively). In fact, the available evidence supporting the notion that reduced meal frequency enhances the efficiency at which fat is deposited derives from studies where reduction in meal frequency was achieved by force-

Table 4

State 3 and 4 mitochondrial respiration in liver homogenates and isolated mitochondria after 1 and 2 weeks of refeeding

\begin{tabular}{|c|c|c|c|c|c|c|c|c|}
\hline & \multicolumn{4}{|c|}{ Homogenate } & \multicolumn{4}{|c|}{ Isolated mitochondria } \\
\hline & \multicolumn{2}{|c|}{$1 \mathrm{wk}$} & \multicolumn{2}{|c|}{$2 \mathrm{wk}$} & \multicolumn{2}{|c|}{$1 \mathrm{wk}$} & \multicolumn{2}{|c|}{$2 \mathrm{wk}$} \\
\hline & Control & Refed & Control & Refed & Control & Refed & Control & Refed \\
\hline \multicolumn{9}{|c|}{ Glutamate + malate } \\
\hline State 3 & $7676.0 \pm 285.8$ & $7965.3 \pm 237.7$ & $7351.3 \pm 447.8$ & $7057.7 \pm 286.3$ & $104.7 \pm 6.1$ & $125.2 \pm 3.4^{*}$ & $128.2 \pm 6.5$ & $119.1 \pm 5.0$ \\
\hline State 4 & $663.8 \pm 39.3$ & $590.8 \pm 25.6$ & $575.5 \pm 41.0$ & $697.2 \pm 56.9$ & $11.6 \pm 0.9$ & $13.3 \pm 0.3$ & $13.5 \pm 0.6$ & $12.3 \pm 0.9$ \\
\hline RCR & $11.6 \pm 0.9$ & $13.6 \pm 0.8$ & $12.9 \pm 0.7$ & $10.4 \pm 0.7$ & $9.7 \pm 0.6$ & $9.1 \pm 0.4$ & $9.6 \pm 0.7$ & $10.0 \pm 0.9$ \\
\hline \multicolumn{9}{|c|}{ Palmitoyl-CoA + carnitine + malate } \\
\hline State 3 & $6074.0 \pm 410.9$ & $6399.0 \pm 246.5$ & $5624.8 \pm 143.6$ & $5562.5 \pm 228.0$ & $95.2 \pm 5.4$ & $110.6 \pm 1.7^{*}$ & $112.2 \pm 8.0$ & $107.6 \pm 7.5$ \\
\hline State 4 & $679.2 \pm 30.5$ & $687.0 \pm 30.4$ & $598.6 \pm 34.6$ & $614.8 \pm 29.4$ & $13.7 \pm 0.5$ & $15.1 \pm 1.0$ & $15.3 \pm 0.9$ & $15.4 \pm 10.6$ \\
\hline RCR & $8.9 \pm 0.7$ & $9.4 \pm 0.5$ & $9.5 \pm 0.4$ & $9.2 \pm 0.6$ & $7.3 \pm 0.5$ & $7.2 \pm 0.4$ & $7.4 \pm 0.6$ & $7.1 \pm 0.7$ \\
\hline
\end{tabular}

Values are the means \pm SEM of 6 different experiments and are expressed as nanogram atoms oxygen/(minute $\times$ milligram protein) for isolated mitochondria and as nanogram atoms oxygen/(minute $\times$ gram wet liver) for homogenate.

$* P<.05$ compared with control (2-way ANOVA followed by Bonferroni posttest). 

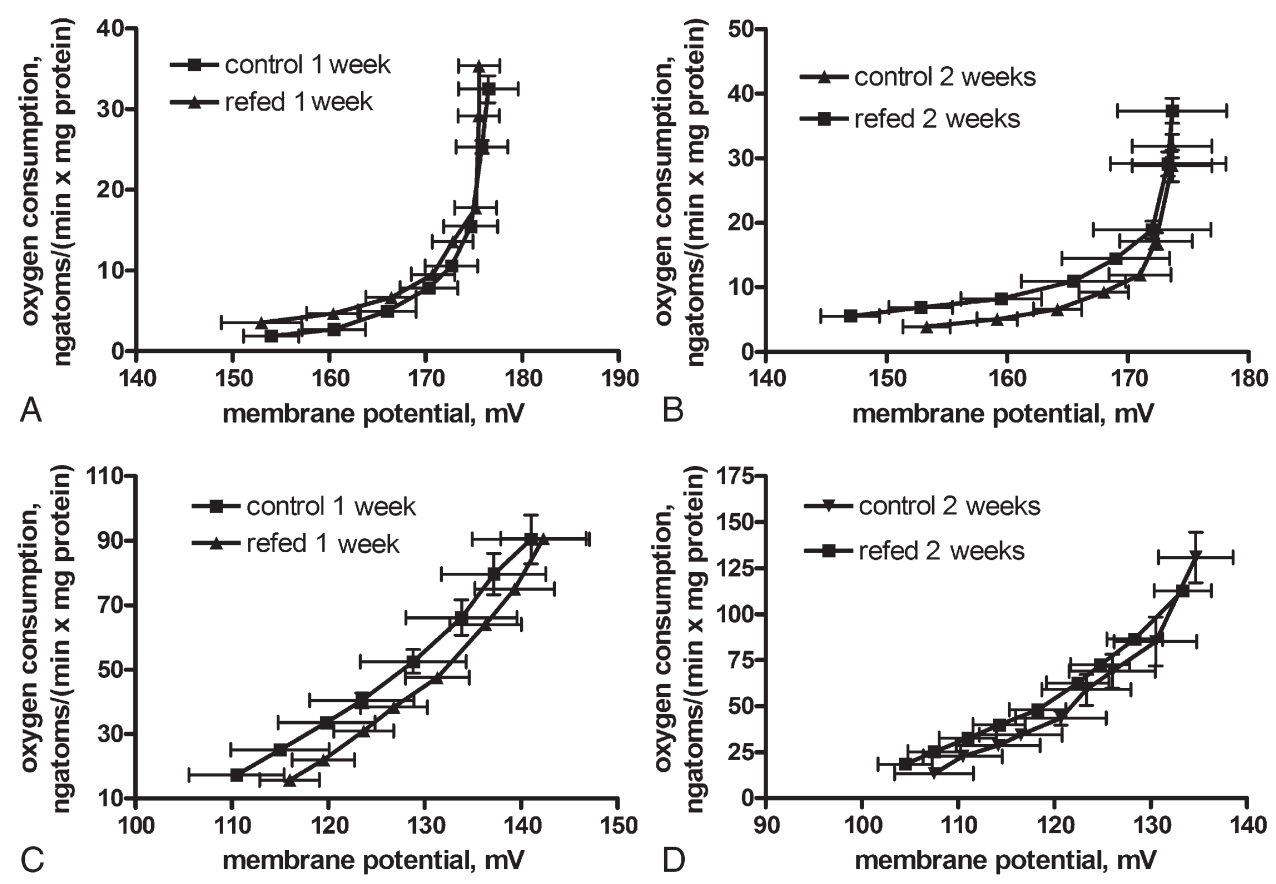

Fig. 3. Basal (A, B) and palmitate-induced (C, D) proton leak kinetics in liver mitochondria after 1 and 2 weeks of refeeding. Results are the means \pm SEM of 6 different experiments.

feeding using gastric intubation $[18,19]$. In contrast, altered meal frequency in rats allowed to eat freely does not alter energy expenditure or fat deposition [20]. Furthermore, in the context of our study, the quantitative contribution of meal pattern seems small for several reasons: $(a)$ the gorging behavior is only present during the first few days of refeeding, yet the rate of catch-up fat is still higher than controls during the second wk of refeeding; and $(b)$ our liver measurements during catch-up fat were made in tissues harvested after this period of gorging behavior, namely, on days 7 and 14 of refeeding.

Further metabolic characterization of refed rats was obtained by measuring 24-hour $\mathrm{VO}_{2}$ and $\mathrm{VCO}_{2}$, which also

Table 5

Total aconitase, active aconitase, active to total aconitase ratio, lipid peroxidation, and SOD activity in liver mitochondria after 1 and 2 weeks of refeeding

\begin{tabular}{lcclcc}
\hline & \multicolumn{2}{c}{1 wk } & & \multicolumn{2}{c}{2 wk } \\
\cline { 2 - 3 } \cline { 5 - 6 } & Control & Refed & & Control & Refed \\
\hline Total aconitase & $27.5 \pm 1.5$ & $30.1 \pm 2.1$ & & $23.0 \pm 2.2$ & $26.7 \pm 2.9$ \\
Active aconitase & $7.3 \pm 0.2$ & $8.8 \pm 0.5$ & & $7.3 \pm 0.3$ & $8.0 \pm 0.3$ \\
Active/total & $0.27 \pm 0.01$ & $0.30 \pm 0.03$ & & $0.33 \pm 0.03$ & $0.31 \pm 0.03$ \\
$\quad$ aconitase & & & & & \\
Lipid peroxidation & $0.81 \pm 0.08$ & $1.06 \pm 0.18$ & & $1.05 \pm 0.06$ & $0.92 \pm 0.08$ \\
SOD activity & $15.0 \pm 1.6$ & $22.8 \pm 2.2^{*}$ & & $24.9 \pm 2.3$ & $19.7 \pm 2.8$ \\
\hline
\end{tabular}

Values are the means \pm SEM of 6 different experiments. Aconitase activity is expressed as milliunits per milligram protein, lipid peroxidation is expressed as nanomoles thiobarbituric acid reactive substances per milligram protein, and SOD activity is expressed as units per milligram protein.

$* P<.05$ compared with control (2-way ANOVA followed by Bonferroni posttest). allow the calculation of NPRQ. After week 1 and week 2 of refeeding, significantly lower values of $\mathrm{VO}_{2}$ and $\mathrm{VCO}_{2}$ were found in refed rats compared with controls, whereas NPRQ values were significantly higher in refed rats than in controls. The lower values of $\mathrm{VO}_{2}$ and $\mathrm{VCO}_{2}$ observed at the end of the second week of refeeding suggest that the lower energy expenditure of refed rats may persist beyond 2 weeks of refeeding under conditions of our studies. In addition, the findings from indirect calorimetry of NPRQ values greater than 1 suggest that the increased lipid deposition typical of refed rats is partly the result of the occurrence of a higher rate of de novo lipogenesis in refed rats than in controls. This result is well in agreement with the "glucose redistribution hypothesis" [7,21], according to which the insulin-resistant state of skeletal muscle found in refed rats is of central importance in sparing glucose that can be diverted toward de novo lipogenesis and fat storage in adipose tissue. Indeed, a higher FAS activity - and hence de novo lipogenic capacity - has been reported in white adipose tissues of refed rats relative to controls [7,21]. Given the importance of the liver a site for de novo lipogenesis, our results showing that hepatic FAS activity is also significantly higher after 1 week of refeeding suggest that the liver could also be contributing importantly to the enhanced whole-body de novo lipid synthesis in refed rats. It is likely that the newly synthesized lipids in the liver are then exported to white adipose tissue for storage - a contention that is consistent with our findings of an enhanced hepatic FAS activity without an increase in hepatic lipid content at week 1 of refeeding. It is also apparent that hepatic FAS activity is no longer elevated at week 2 of refeeding despite the 
persistently lower 24-hour energy expenditure and higher whole-body de novo lipogenesis (as assessed by indirect calorimetry) at this time point. One explanation for this apparent discrepancy between changes in liver FAS activity and whole-body lipogenesis during catch-up fat might reside in the possibility that, whereas both liver and adipose tissue contribute to de novo lipogenesis during the earlier phase of catch-up fat, it is only adipose tissue that remains an important site of enhanced de novo lipogenesis in the later phase of catch-up fat. The relative contribution of liver and adipose tissue to de novo lipogenesis during catch-up fat is certainly an important area for future research.

\subsection{Hepatic mitochondrial mass and density}

In our previous studies carried out in skeletal muscle, we showed a decrease in the mass and the activity of subsarcolemmal mitochondria in this tissue at week 1 of refeeding [6]. The studies reported here also indicate a significant decrease in hepatic mitochondrial mass at week 1 of refeeding. These changes in hepatic mitochondrial mass therefore parallel those found in skeletal muscle at similar time points from the same rat model of catch-up fat [6], thereby suggesting that down-regulation of mitochondrial mass may be a general response of tissues to energy deprivation. Opposite changes (ie, increases) in hepatic mitochondrial mass have been found in conditions of excess energy supply, such as long-term high-fat feeding [22], thereby underscoring a strong correlation between mitochondrial mass and cellular energy availability. Given our other findings here that hepatic mitochondrial numerical density is not different between refed and control animals, the decrease in hepatic mitochondrial mass may thus be the result of a general down-regulation of the energy-consuming pathway of protein synthesis rather than that of a decrease in mitochondrial biogenesis.

\subsection{Hepatic mitochondrial respiratory capacity}

In skeletal muscle, the decrease in mitochondrial protein mass was found to be accompanied by a decrease in organelle respiratory function, whereas at the hepatic level, the potential impact on cellular energy utilization of the decreased mitochondrial protein mass that occurs at week 1 of refeeding is counteracted by a concomitant increase in specific respiratory capacities. In fact, state 3 respiration with NAD- and FAD-linked substrates was significantly higher in isolated mitochondria. In liver mitochondria, adenine nucleotide carrier and adenosine triphosphate synthase are not significant control steps for glutamate- and palmitoylCoA-supported respiration [23]. Consequently, the increased state 3 respiration found in hepatic mitochondria could be attributed to changes in the rate-limiting steps common to the oxidation of both substrates, that is, the activity of the respiratory chain from complex I onward. The increased respiratory capacity found in isolated mitochondria may have relevance in sustaining cellular adenosine triphosphate production rate, a fundamental step needed to support the energy-consuming machinery of hepatic lipogenesis of refed rats.

\subsection{Hepatic mitochondrial proton leak and oxidative stress}

An additional mechanism that can regulate mitochondrial energy production is the degree of coupling of oxidative phosphorylation, which in turn depends on mitochondrial inner membrane permeability to protons. However, our measurements of both basal and fatty acid-induced mitochondrial proton leak kinetics in mitochondria from refed and control rats did not reveal any between-group differences in these parameters. This result showing no change in hepatic mitochondrial efficiency in the refed group can be considered representative of the "in vivo" condition. In fact, another important factor that could induce an increase in mitochondrial efficiency is a decrease in the cellular concentration of free fatty acids. Because in refed rats hepatic lipogenesis is increased, it is very unlikely that there is a decrease in cellular free fatty acid concentration. In addition, we did not observe any difference in aconitase activity and lipid peroxidation, 2 markers of oxidative damage, which in turn is generally correlated with mitochondrial function. After 1 week of refeeding, the lack of oxidative damage is in agreement with the enhanced antioxidant defense (increased SOD activity) and with the increase of hepatic FAS activity, an energetically expensive pathway. These findings in the liver contrast with those previously obtained in skeletal muscle mitochondria, where an increased oxidative damage has been found during refeeding [6].

\section{Conclusions}

In conclusion, our results indicate that fat storage after caloric restriction is characterized by robust changes in lipid balance and marked stimulation of the pathway of de novo lipogenesis even in the absence of hyperphagia. Integrating our results here in liver with those previously reported in skeletal muscle, it appears that liver and skeletal muscle both contribute to catch-up fat via different pathways. Skeletal muscle, through a reduction in mass and respiratory activity of subsarcolemmal mitochondria [6], could be contributing directly to the suppressed thermogenesis that favors glucose sparing for catch-up fat. The liver, by contrast, through enhanced hepatic state 3 mitochondrial respiration and increased FAS activity, might be contributing to catch-up fat via an enhancement in its machinery for de novo lipogenesis. The orchestration of catch-up fat through suppressed thermogenesis (resulting in glucose sparing) in skeletal muscle and the concomitant enhancement of (glucose-derived) de novo lipogenesis in liver could have important implications both to the ease of obesity relapse after slimming and to the predisposition of catch-up fat during catch-up growth to increased risk for obesity and diabetes. 


\section{Acknowledgment}

This work was supported by a grant from University "Federico II" of Naples and by the Swiss National Research Foundation (grant 32003B-113634). The authors thank Dr Emilia De Santis for skilful management of animal house.

\section{References}

[1] Ramsey JJ, Harper ME, Weindruch R. Restriction of energy intake, energy expenditure and aging. Free Rad Biol Med 2000;29:946-68.

[2] Dulloo AG, Girardier L. Adaptive changes in energy expenditure during refeeding following low calorie intake: evidence for a specific metabolic component favouring fat storage. Am J Clin Nutr 1990;52:415-20.

[3] Dulloo AG, Jacquet J, Montani JP. Pathways from weight fluctuation to metabolic disease: focus on maladaptive thermogenesis during catch-up fat. Int J Obes 2002;26(Suppl 2):S46-57.

[4] Crescenzo R, Samec S, Antic V, Rohner-Jeanrenaud F, Seydoux J, Montani JP, et al. A role for suppressed thermogenesis favouring catch-up fat in the pathophysiology of catch-up growth. Diabetes 2003;52:1090-7.

[5] Rolfe DFS, Brown GC. Cellular energy utilization and molecular origin of standard metabolic rate in mammals. Physiol Rev 1997;77: 731-58.

[6] Crescenzo R, Lionetti L, Mollica MP, Ferraro M, D'Andrea E, Mainieri D, et al. Altered skeletal muscle subsarcolemmal mitochondrial compartment during catch-up fat after caloric restriction. Diabetes 2006;55:2286-93.

[7] Cettour-Rose P, Samec S, Russell AP, Summermatter S, Mainieri D, Carrillo-Theander C, et al. Redistribution of glucose from skeletal muscle to adipose tissue during catch-up fat. A link between catch-up growth and later metabolic syndrome. Diabetes 2005;54:751-6.

[8] Folch J, Lees M, Stanley GHS. A simple method for the isolation and purification of total lipids from animal tissues. $\mathrm{J}$ Biol Chem 1957;226:497-510.

[9] Penicaud L, Ferre P, Assimacopoulos-Jeannet F, Perdereau D, Leturque A, Jeanrenaud B, et al. Increased gene expression of lipogenic enzymes and glucose transporter in white adipose tissue of suckling and weaned obese Zucker rats. Biochem J 1991;279:303-8.

[10] Iossa S, Lionetti L, Mollica MP, Barletta A, Liverini G. Energy intake and utilization vary during development in rats. J Nutr 1999;129: 1593-6.

[11] Gundersen HJ, Bendtsen TF, Korbo L, Marcussen N, Møller A, Nielsen K, et al. Some new, simple and efficient stereological methods and their use in pathological research and diagnosis. APMIS 1988;96:379-94.

[12] Srere PA. Citrate synthase. Meth Enzymol 1969;13:3-5.

[13] Iossa S, Lionetti L, Mollica MP, Barletta A, Liverini G. Effect of highfat feeding on metabolic efficiency and mitochondrial oxidative capacity in adult rats. Br J Nutr 2003;90:953-60.

[14] Estabrook RW. Mitochondrial respiratory control and the polarographic measurement of ADP:O ratios. Meth Enzymol 1967;10 41-7.

[15] Fernandes MA, Custodio JBA, Santos MS, Moreno AJ, Vicente JA. Tetrandrine concentrations not affecting oxidative phosphorylation protect rat liver mitochondria from oxidative stress. Mitochondrion 2006;6:176-85.

[16] Lionetti L, Mollica MP, Crescenzo R, D’Andrea E, Ferraro M, Bianco $\mathrm{F}$, et al. Skeletal muscle subsarcolemmal mitochondrial dysfunction in high-fat fed rats exhibiting impaired glucose homeostasis. Int J Obes 2007;31:1596-604.

[17] Elia M, Livesey G. Theory and validity of indirect calorimetry during net lipid synthesis. Am J Clin Nutr 1988;47:591-607.

[18] Cohn C, Joseph D. Changes in body composition with force-feeding. Am J Physiol 1959;196:965-8.

[19] Rothwell NJ, Stock MJ. A paradox in the control of energy intake in the rat. Nature 1978;273:146-7.

[20] Hill JO, Anderson JC, Lin D, Yakubu F. Effects of meal frequency on energy utilization in rats. Am J Physiol 1988;255:R616-621.

[21] Dulloo AG. Thrifty energy metabolism in catch-up growth trajectories to insulin and leptin resistance. Best Pract Clin Endocrinol Metab 2008;22:155-71.

[22] Crescenzo R, Bianco F, Falcone I, Prisco M, Liverini G, Iossa S. Alterations in hepatic mitochondrial compartment in a model of obesity and insulin resistance. Obesity 2008;16:958-64.

[23] Liverini G, Goglia F, Lanni A, Iossa S, Barletta A. Elevated hepatic mitochondrial oxidative capacities in cold exposed rats. Comp Biochem Physiol 1990;97B:327-31. 